\title{
Antibody-mediated procoagulant platelets in SARS-CoV-2-vaccination associated immune thrombotic thrombocytopenia
}

Haematologica 2021

Volume 106(8):2170-2179

\section{Correspondence:}

TAMAM BAKCHOUL

tamam.bakchoul@med.uni-tuebingen.de

FALKO FEND

falko.fend@med.uni-tuebingen.de

Received: April 16, 2021.

Accepted: May 11, 2021.

Pre-published: May 20, 2021.

https://doi.org/10.3324/haematol.2021.279000

(C)2021 Ferrata Storti Foundation

Material published in Haematologica is covered by copyright. All rights are reserved to the Ferrata Storti Foundation. Use of published material is allowed under the following terms and conditions:

https://creativecommons.org/licenses/by-nc/4.0/legalcode. Copies of published material are allowed for personal or internal use. Sharing published material for non-commercial purposes is subject to the following conditions:

https://creativecommons.org/licenses/by-nc/4.0/legalcode, sect. 3. Reproducing and sharing published material for commercial purposes is not allowed without permission in writing from the publisher.

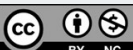

Karina Althaus, ${ }^{1,2^{*}}$ Peter Möller, ${ }^{3 *}$ Günalp Uzun, ${ }^{2}$ Anurag Singh, ${ }^{1}$ Annika Beck, ${ }^{3}$ Martin Bettag, ${ }_{4}^{4}$ Hans Bösmüller, ${ }^{5}$ Martina Guthoff, ${ }^{6}$ Franziska Dorn, ${ }^{7}$ Gabor C. Petzold, ${ }^{8}$ Hans Henkes, ${ }^{9}$ Nils Heyne, ${ }^{6}$ Hassan Jumaa, ${ }^{10}$ Kornelia Kreiser, ${ }^{3}$ Caroline Limpach, ${ }^{11}$ Beate Luz, ${ }^{12}$ Matthias Maschke, ${ }^{13}$ Janis A. Müller, ${ }^{14}$ Jan Münch, ${ }^{14}$ Simon Nagel, ${ }^{15}$ Bernd Pötzsch, ${ }^{16}$ Jens Müller, ${ }^{16}$ Christoph Schlegel, ${ }^{17}$ Andreas Viardot, ${ }^{18}$ Hansjörg Bäzner, ${ }^{19}$ Marc Wolf, ${ }^{19}$ Lisann Pelzl, ${ }^{1}$ Verena Warm, ${ }^{5}$ Winfried A. Willinek, ${ }^{20}$ Jochen Steiner, ${ }^{21}$ Nicole Schneiderhan-Marra, ${ }^{22}$ Dominik Vollherbst, ${ }^{15}$ Ulrich J. Sachs, ${ }^{23}$ Falko Fend ${ }^{5 \#}$ and Tamam Bakchoul ${ }^{1,2 \#}$

${ }^{1}$ Institute for Clinical and Experimental Transfusion Medicine, Medical Faculty of Tübingen, Tübingen University Hospital, Tübingen; ${ }^{2}$ Center for Clinical Transfusion Medicine, Tübingen University Hospital, Tübingen; ${ }^{3}$ Institute for Pathology, UIm University Hospital, UIm; ${ }^{4}$ Department of Neurosurgery, Krankenhaus der Barmherzigen Brüder Trier, Trier; ${ }^{5}$ Institute for Pathology and Neuropathology, Tübingen University Hospital, Tübingen; ${ }^{6}$ Department of Internal Medicine IV, Section of Nephrology and Hypertension, Tübingen University Hospital, Tübingen; 'Department of Neuroradiology, Bonn University Hospital, Bonn; ${ }^{8}$ Section of Vascular Neurology, University Hospital Bonn; ' ${ }^{2}$ epartment of Neuroradiology, Klinikum Stuttgart, Stuttgart; ${ }^{10}$ Institute for Immunology, UIm University Hospital, UIm; ${ }^{11}$ Department of Neurology, Krankenhaus der Barmherzigen Brüder Trier, Trier; ${ }^{12}$ Institute of Transfusion Medicine, Klinikum Stuttgart, Stuttgart; ${ }^{13}$ Department of Neurology, Krankenhaus der Barmherzigen Brüder Trier, Trier; ${ }^{14}$ Institute of Molecular Virology, Ulm University Medical Center, UIm; ${ }^{15}$ Department of Neuroradiology, Heidelberg University Hospital, Heidelberg; ${ }^{16}$ Institute for Experimental Hematology and Transfusion Medicine, Bonn; ${ }^{17} \mathrm{UIm}$ University Hospital, UIm; ${ }^{18} \mathrm{Internal}$ Medicine III, UIm University Hospital, Ulm; ${ }^{19}$ Department of Neurology, Klinikum Stuttgart, Stuttgart; ${ }^{20}$ Department of Radiology, Krankenhaus der Barmherzigen Brüder Trier, Trier; ${ }^{21}$ Anesthesiology and Intensive Care Medicine, Tübingen University Hospital, Tübingen; ${ }^{22}$ Natural and Medical Sciences Institute, University of Tübingen, Reutlingen and ${ }^{23}$ Department of Thrombosis and Hemostasis and Institute of Immunology and Transfusion Medicine, Giessen, Germany

* $K A$ and $P M$ contributed equally as co-first authors.

${ }^{*} F F$ and TB contributed equally as co-senior authors.

\section{ABSTRACT}

The COVID-19 pandemic has resulted in significant morbidity and mortality worldwide. In order to prevent severe infection, mass COVID-19 vaccination campaigns with several vaccine types are currently underway. We report pathological and immunological findings in eight patients who developed vaccine-induced immune thrombotic thrombocytopenia (VITT) after administration of SARS-CoV-2 vaccine ChAdOx1 $\mathrm{nCoV}-19$. We analyzed patient material using enzyme immune assays, flow cytometry and heparin-induced platelet aggregation assay and performed autopsies on two fatal cases. Eight patients (five females, three males) with a median age of 41.5 years (range, 24-53) were referred to us with suspected thrombotic complications 6 to 20 days after ChAdOx1 nCoV-19 vaccination. All patients had thrombocytopenia at admission. Patients had a median platelet count of $46.5 \times 10^{9} / \mathrm{L}$ (range, 8-92). Three had a fatal outcome and five were successfully treated. Autopsies showed arterial and venous thromboses in various organs and the occlusion of glomerular capillaries by hyaline thrombi. Sera from VITT patients contain high-titer antibodies against platelet factor 4 (PF4) (optical density [OD] 2.59 \pm 0.64 ). PF4 antibodies in VITT patients induced significant increase in procoagulant markers ( $\mathrm{P}$ selectin and phosphatidylserine externalization) compared to healthy volunteers and healthy vaccinated volunteers. The generation of procoagulant platelets was PF4 and heparin dependent. We demonstrate the contribution of antibody-mediated platelet activation in the pathogenesis of VITT. 


\section{Introduction}

COVID-19 infection has resulted in considerable morbidity and mortality in the last 15 months. ${ }^{1}$ Within an exceptionally short time, several SARS-CoV-2 vaccines have been licensed and used worldwide. ${ }^{2}$ Safety signals have been, however, noted. Center for Disease Control and Prevention (CDC) in the US reported in the beginning of March 26 cases of venous thromboembolism, 20 cases of thrombosis and 41 ischemic strokes in individuals vaccinated with mRNA vaccines in the US. More than 200 cases with thrombosis among 34 million persons vaccinated with ChAdOx1 nCoV-19 have been reported to the European database of suspected adverse reactions, EudraVigilance. After the investigation of reported cases, European Medical Association (EMA) found a link between ChAdOx1 nCoV-19 and unusual thrombotic events and concomitant thrombocytopenia. Although the World Health Organization (WHO) and EMA concluded that the benefit of vaccination with ChAdOx1 nCoV-19 outweighs the risks associated with thrombosis and thrombocytopenia, several countries instituted restrictions on the use of ChAdOx1 nCoV-19. The unusual clinical constellation of cerebral venous sinus thrombosis (CVST) and thrombocytopenia is called vaccine-induced immune thrombotic thrombocytopenia (VITT). We studied eight cases with thrombocytopenia and primarily with suspected CVST but also other thromboembolic complications to better understand the pathophysiology of VITT. In this study, we identified antibody-mediated procoagulant platelets as a novel mechanism associated with VITT.

\section{Methods}

\section{Study cohort and evaluation of the clinical data}

Eight patients were referred to different university hospitals with neurological or hematological symptoms after vaccination with ChAdOx1 nCoV-19 (AstraZeneca, London, UK) between February $1^{\text {st }}$ and April $6^{\text {th }} 2021$. Six patients admitted to university hospitals and two patients, who were initially admitted to local hospitals, were later transferred to university hospitals. Medical records were used to collect treatments and outcome. Diagnosis of thromboembolic complications was made when indicated by clinical or laboratory findings and/or based on computed tomography, ultrasound imaging or in case of death by autopsy.

Blood samples were collected to exclude heparin-induced thrombocytopenia (HIT). Blood samples from non-vaccinated healthy blood donors $(n=24,17$ females, mean age $36.1 \pm 13.7$ years) and from healthy vaccinated before and after the first vaccination with ChAdOx1 $\mathrm{nCoV}-19$ ( $\mathrm{n}=41,29$ females, mean age $37.3 \pm 10.9$ years) served as healthy controls.

In addition, sera from 29 COVID-19 patients who had serial HIT immunoglobulin G (IgG)-enzyme immune assay (EIA) measurements during hospitalization were also included in the study (seven females, mean age $65.3 \pm 14.1$ years). Clinical data from the ICU COVID-19 patients and a VITT-patient (case \#7) were reported in previous studies. ${ }^{3,4}$

\section{Bead-based multiplex assay for detection of COVID-19 antibodies}

COVID-19 antibodies were measured with a multiplex assay (NMI, Reutlingen, Germany) with the FLEXMAP 3D ${ }^{\oplus}$ system (Luminex Corporation, Austin, USA). ${ }^{5}$

\section{Testing for anti-PF4/heparin antibodies}

A commercially available EIA was used in accordance with the manufacturer's instructions (Hyphen Biomed, Neuville-sur-Oise, France). The ability of sera to activate platelets was tested using the functional assay heparin-induced platelet aggregation assay (HIPA) as previously described. ${ }^{6}$ For more details, see the Online Supplementary Appendix.

\section{Serological characterization of PF4 antibody}

Antibody binding to PF4 and the receptor binding domain of Spike protein (Spike-RBD and S2 domain) was analyzed using an in-house EIA.

\section{Assessment of antibody-mediated procoagulant platelets}

Patients' sera were incubated with washed platelets $\left(7.5 \times 10^{\circ}\right)$ for $1.5 \mathrm{~h}^{*}$ under different conditions at room temperature. Platelets were then stained with Annexin V-FITC and CD62-APC (Immunotools, Friesoythe Germany) and directly analyzed by flow cytometry (FC). For more details see the Online Supplementary Appendix.

\section{Ethics statement}

The study was conducted in accordance with the Declaration of Helsinki. The study protocol was approved by the Institutional Review Board of the University of Tuebingen $(236 / 2021 \mathrm{BO} 2,224 / 2021 \mathrm{BO} 2)$ and analysis of sera from $\mathrm{ChAdOx} 1 \mathrm{nCoV}-19$ vaccinated individuals were performed at the University of Ulm (99/21).

\section{Statistical analyses}

The statistical analysis was performed using GraphPad Prism, Version 7.0 (GraphPad, La Jolla, USA). Since potential daily variations in FC measurements might result in bias in data analysis, test results were normalized to two healthy donors tested in parallel at the same time point. Data in the text are presented as median (range), mean \pm standard deviation (SD) or numbers ( $n$ in \%).

\section{Data sharing statement}

Data may be requested for academic collaboration from the corresponding author.

\section{Results}

\section{Clinical and laboratory features of vaccine-induced} immune thrombotic thrombocytopenia

Eight patients (five females, three males) with a median age of 41.5 years (range, 24-53) were referred with suspected thrombotic complications after ChAdOx1 nCoV19 vaccination. Demographic data are summarized in Table 1 . The patients were admitted to hospital 6 to 20 days after ChAdOx1 nCoV-19 vaccination. All patients had thrombocytopenia at admission with a median platelet count of $46.5 \times 10^{9} / \mathrm{L}$ (range, 8-92). D-dimer was available in five patients, which was $9 \mu \mathrm{g} / \mathrm{mL}$ or higher. Thrombosis was detected in six patients at admission and two developed thrombosis during hospitalization (Figure 1). Thrombotic events included cerebral venous sinus thrombosis (five patients), pulmonary embolism (four patients), deep vein thrombosis (one patient), and thrombosis in other organs (three patients). Three of eight patients had more than one thrombotic event. Three patients presented initially with bleeding signs with easy 
bruising and petechiae, which might be an early sign of VITT. One patient underwent a successful thrombus removal by endovascular rheolysis. Three of eight patients died (on day 6 [case \#1], day 10 [case \#2] and day 7 [case \#3] of hospitalization). All surviving patients received anticoagulation. Four patients received intravenous immunoglobulin (IVIG) combined with non-heparin anticoagulation.

\section{Pathological findings}

Autopsy was performed in two of three deceased patients. Autopsy of case \#2 showed complete throm- botic obstruction of the straight, sagittal and transversal cerebral sinuses, subarachnoidal hemorrhage, cerebral edema and bilateral pulmonary embolism in mid-sized arteries and obstruction of glomerular arterioles and capillaries by hyaline microthrombi containing fibrin and platelets (Figure 2A and B). Autopsy of case \#3 showed massive cerebral hemorrhage and cerebral edema, bilateral pulmonary thromboembolism and obstruction of glomeruli by hyaline microthrombi (Figure 2C to G). Histology of the bone marrow was normal in both cases without any hint of increased thrombopoesis.

Table 1. Demographic and clinical data of cases with vaccine induced immune thrombotic thrombocytopenia.

\begin{tabular}{|c|c|c|c|c|c|c|c|c|c|c|}
\hline Case \# & Age & Sex & $\begin{array}{l}\text { First symptoms } \\
\text { after vaccination } \\
\text { (days) }\end{array}$ & $\begin{array}{l}\text { Thrombosis/ } \\
\text { Bleeding }\end{array}$ & $\begin{array}{l}\text { Thrombotic } \\
\text { risk factors }\end{array}$ & $\begin{array}{c}\text { PLT, } \\
\left(150-450 \times 10^{9} / \mathrm{L}\right)\end{array}$ & $\begin{array}{c}\text { D-Dimer, } \\
(<0.5 \mu \mathrm{g} / \mathrm{mL})\end{array}$ & $\begin{array}{l}\text { Fhrinogen, } \\
\text { (170-410 mg/dL) }\end{array}$ & INR & aPTा, (>40s) \\
\hline 1 & 47 & $\mathrm{f}$ & 7 & CVST & none & 10 & $>35$ & 128 & 1.30 & 23 \\
\hline 2 & 48 & $\mathrm{f}$ & 6 & CVST, PE & n.a. & 40 & n.a. & n.a. & 1.16 & 22.9 \\
\hline 3 & 24 & $\mathrm{~m}$ & 10 & $\begin{array}{l}\text { bleeding, } \\
\text { multiple } \\
\text { thrombosis }\end{array}$ & $\begin{array}{l}\text { heterozygous } \\
\text { FVL mutation }\end{array}$ & 22 & n.a. & 109 & 1.20 & 42 \\
\hline 4 & 53 & $\mathrm{~m}$ & 9 & DVT, PE & none & 8 & $>35$ & 126 & 1.01 & 25 \\
\hline 5 & 47 & $\mathrm{f}$ & 7 & CVST & none & 56 & 9 & 263 & 1.25 & 35 \\
\hline 6 & 32 & $\mathrm{~m}$ & 20 & PE & none & 71 & n.a. & n.a. & n.a. & n.a. \\
\hline 7 & 36 & $\mathrm{f}$ & 17 & CVST & none & 92 & 13 & n.a. & 1.19 & 22 \\
\hline 8 & 29 & $\mathrm{f}$ & 7 & CVST & contra-ception & 53 & 32 & 274 & 1.00 & 23 \\
\hline
\end{tabular}

CVST: indicates cerebral venous sinus thrombosis; DVT: deep vein thrombosis; FVL: FactorV Leiden; n.a.: not available; PE, pulmonary embolism; PLT: platelet.

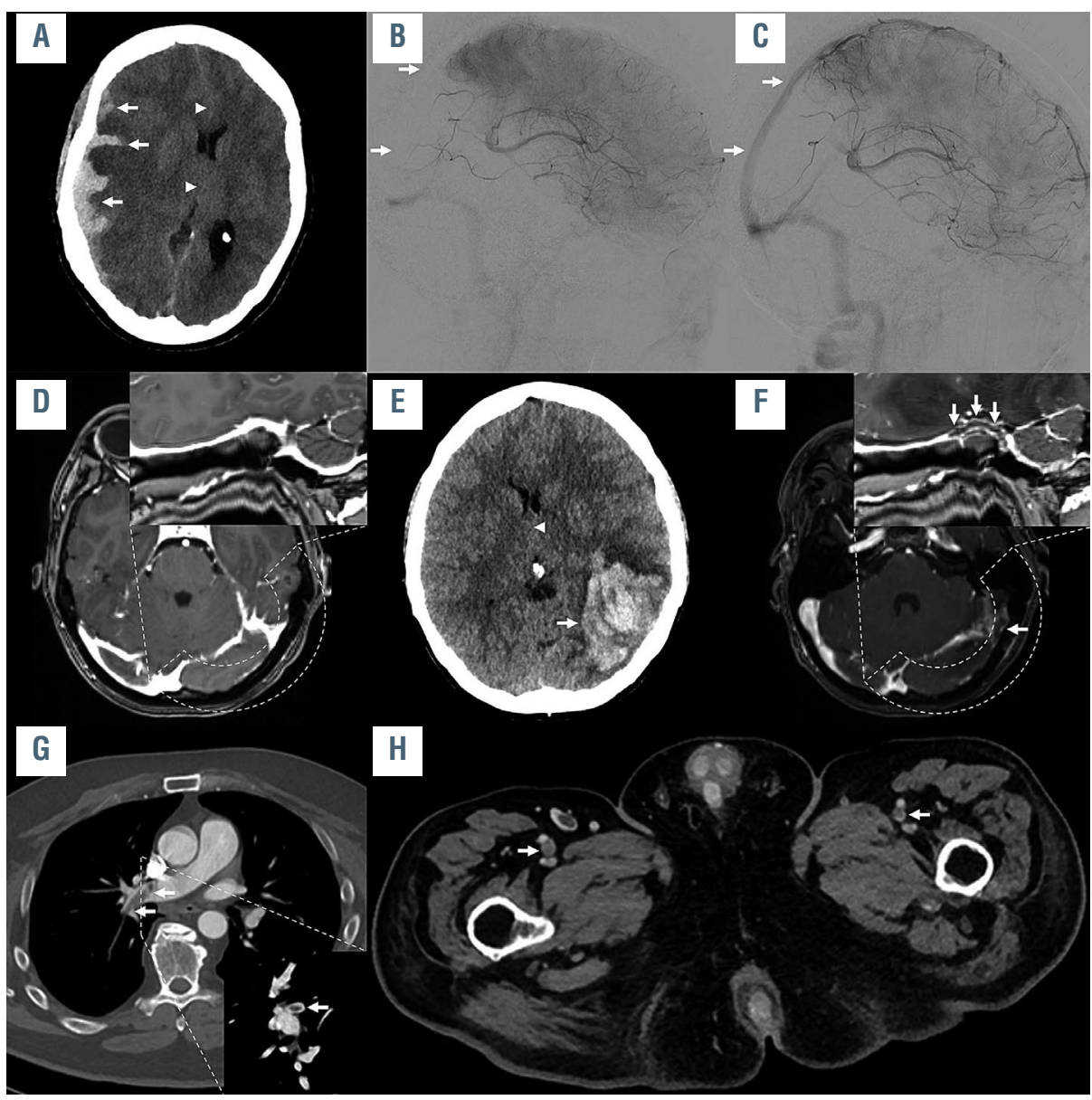

Figure 1. Imaging example of three illustrative cases. Imaging exam ples of case \#1 (A to C), case \#8 (D to $\mathrm{F}$ ) and case \#4 ( $\mathrm{G}$ and $\mathrm{H}$ ). In case \#1, non-enhanced computed tomography imaging (A) showed a parenchymal and subdural hemorrhage (arrows in A), causing a midline shift (arrowheads in A). Digita subtraction angiography was performed (B) showing thrombosis of the right sigmoid and transverse sinus, superior sagittal sinus (arrows in B), and straight sinus. Angiography after mechanica recanalization $(\mathrm{C})$ shows the recanalized cerebral sinuses (superior sagittal sinus marked with arrows). In case \#8, cerebral imaging 7 days after vaccination was unremarkable (curved reconstruction of the left transverse and sigmoid sinus shown in the right upper corner of $D$ and $F$ ). She worsened, which led to a repeated cerebral imaging, showing a large intraparenchymal hemorrhage in the left temporal lobe (arrow in E), causing midline shift (arrowhead in $\mathrm{E}$ ), caused by a thrombosis of the transverse and sigmoid sinus (arrows in $\mathrm{F}$ ), as well as of the adjacent tentorial veins. In case \#4, a thrombus in the right pulmonary artery was observed (arrows in E; coronal reconstruction shown in the right lower corner of G). Further imaging also revealed thrombi in the femora veins on both sides (arrows in $\mathrm{H}$ ). 
Immunoglobulin G binding profile of sera from vaccineinduced immune thrombotic thrombocytopenia

High-titer PF4/heparin antibodies were detected in all sera (eight of eight, 100\%) using the IgG PF4/heparin EIA. Interestingly, binding of all sera was inhibited in the presence of high concentration of heparin (mean optical density [OD] of IgG antibodies against PF4/heparin complexes: $2.591 \pm 0.642$ versus $0.176 \pm 0.073$, respectively, $P<0.0001$, Figure $3 \mathrm{~A})$. No correlation was found between the PF4/heparin antibodies and the detected COVID-19 antibodies in VITT patients and in vaccinated controls (Online Supplementary Figure S1A to D [I-IV]). Among non-vaccinated controls only one subject (4\%) had a PF4/heparin antibodies in EIA (data not shown).

We next investigated the PF4-seroconversion after vaccination with $\mathrm{ChAdOx} 1 \mathrm{nCoV}-19$, as well as during severe SARS-CoV-2 infection (Figure 3B). We found that four of $41(9.8 \%)$ vaccinated healthy individuals and four of 25 (16\%) patients with severe COVID-19 seroconverted with IgG antibodies against PF4/heparin complexes within 14 days (Figure $3 \mathrm{~B}$ ). Next, we tested IgG binding to platelets by FC. An increase in IgG binding to test platelets was observed (fold increase [FI] in mean fluorescence [MF] intensity compared to healthy controls [FI of MFI]: $4.39 \pm 1.15$ vs. $1 \pm 1.10, \quad P=0.026$, Figure 4, Online Supplementary Figure S2A). IgG binding to platelets was inhibited by heparin at high concentrations (FI of MFI IgG binding: $1.51 \pm 0.66, P=0.016$ ), but not at low concentrations (FI of MFI of IgG binding: $3.60 \pm 2.01, P=0.688$ ). Only one serum showed increased binding to platelets in the presence of PF4 and the vaccine ChAdOx1 nCoV-19 (case \#4, Figure 4). Spike-RBD did not induce a significant change in IgG binding in VITT patients (Figure 5A). Similar results were observed when S2 protein was added (Figure $5 \mathrm{~B})$. IgG binding was also observed when sera from ChAdOx1 nCoV-19 vaccinated volunteers with IgG PF4 antibodies were tested (Online Supplementary Figure 2B). However, severe COVID-19 patients with IgG PF4 antibodies showed no increase in IgG binding (Online Supplementary Figure S2C).

\section{The impact of Spike-RBD on the binding of anti-PF4 antibodies}

Compared to healthy controls, sera from VITT patients showed strong binding to PF4 in the in-house EIA (OD IgG antibodies against PF4: $1.03 \pm 0.04$ vs. $0.110 \pm 0.002$, respec-
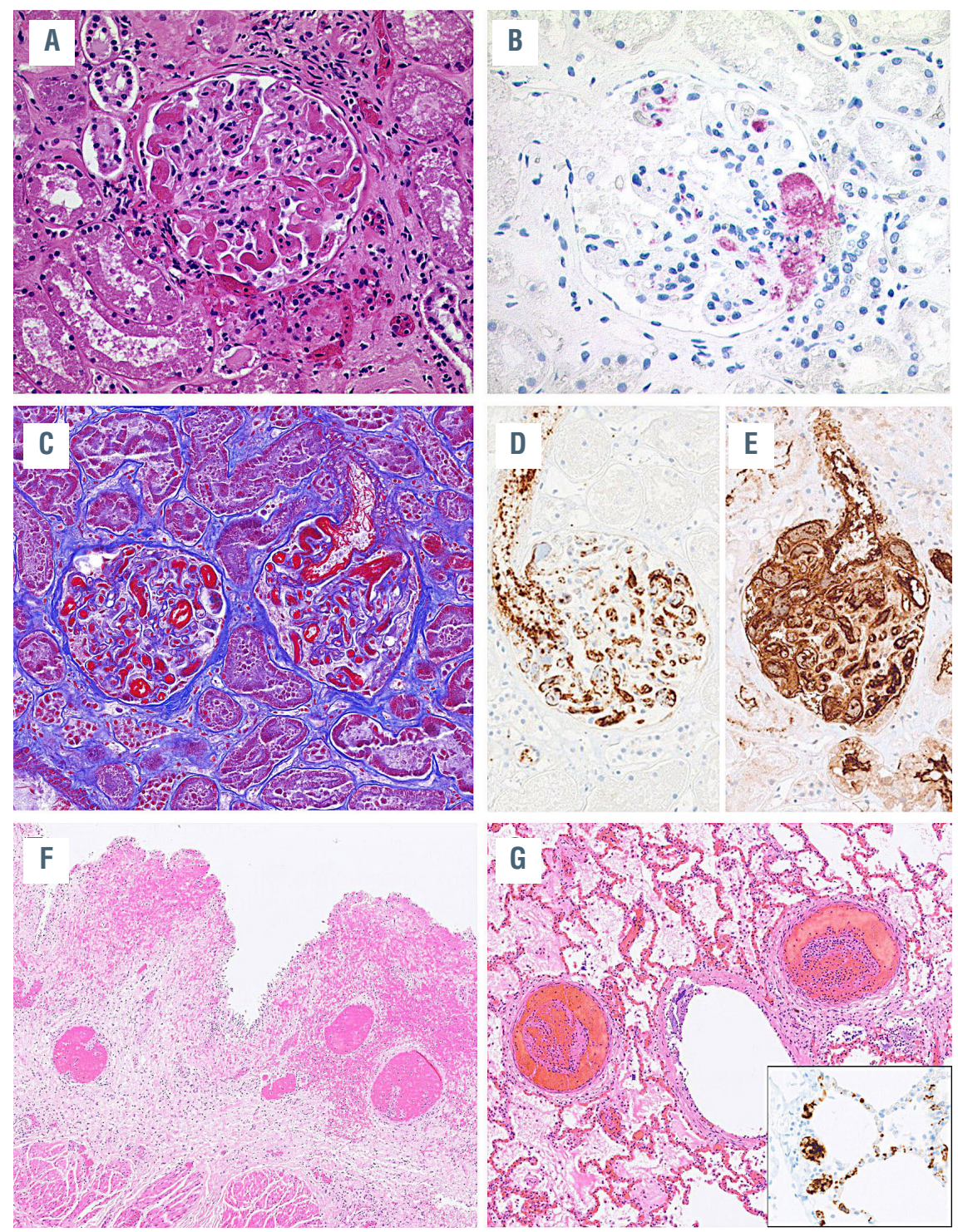

Figure 2. Histopathological findings in case \#2 and case \#3. (A) Case \#2: occlusion of glomerular capillary loops by hyaline thrombi. Hematoxylin-Eosin (H\&E) staining, original magnification 200x. (B) Deposition of platelets in glomerular vessels documented by CD42b staining, immunoperoxidase staining, magnification 200x. (C) Case \#3: occlusion of glomerular capillary loops by hyaline thrombi with fibrin deposits highlighted in red, Masson's trichrome stain, magnification 200x. (D) Immunostaining for CD61 and $(E)$ fibrin demonstrate the massive intravascular deposits of fibrin and platelets, immunoperoxidase staining, magnification 200x. (F) Thrombotic occlusions of submucosal vessels in the urinary bladder with hemorrhage. H\&E staining, magnification 40x. (F) Thrombotic occlusion of medium-sized pulmonary vessels. H\&E stining, magnification 40x. (G) Insert shows platelet deposits in pulmonary capillaries stained for CD61, immunoperoxidase staining, magnification 400x. 


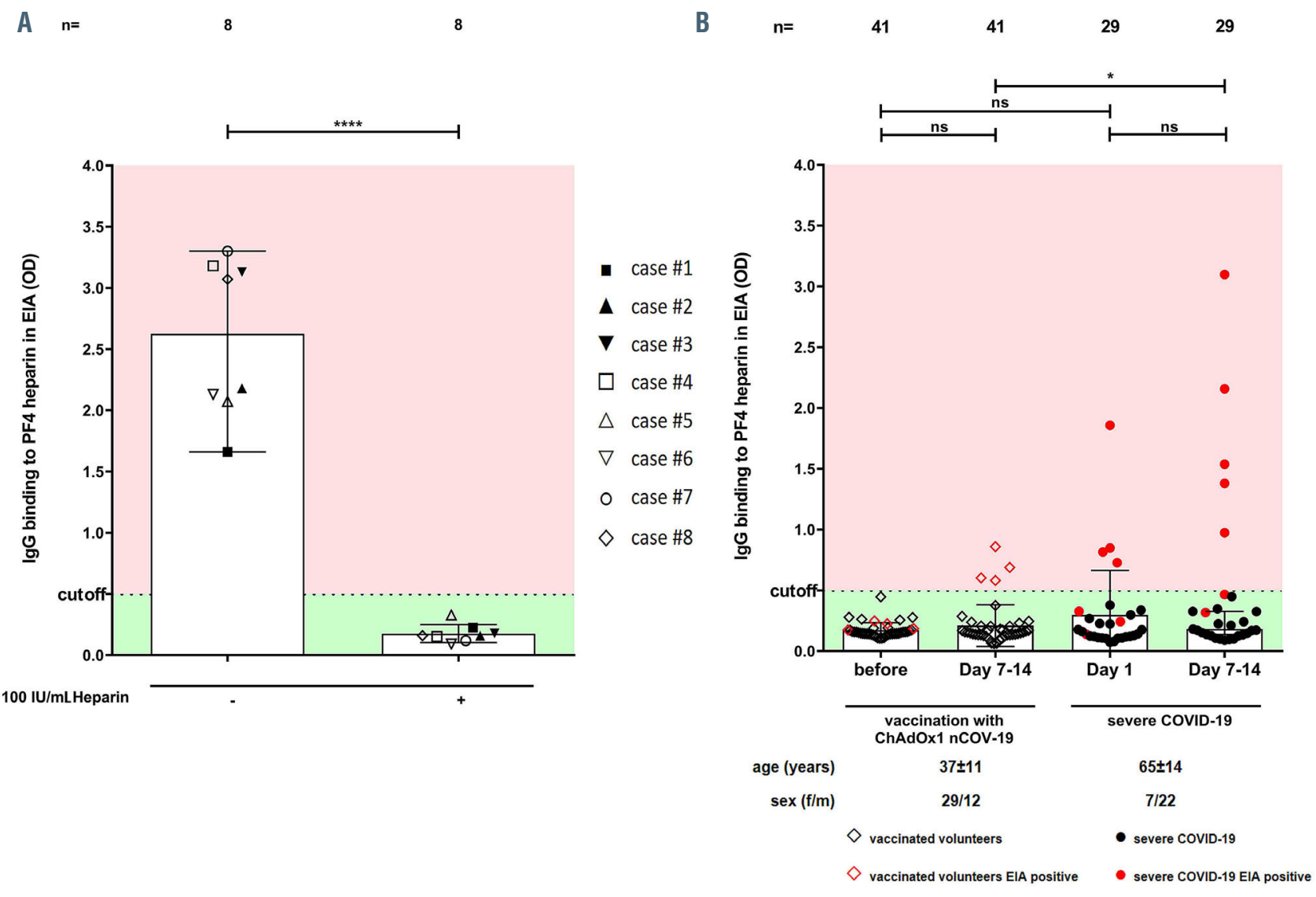

Figure 3. Binding profile of sera from vaccine-induced immune thrombotic thrombocytopenia. (A) Results of the PF4/heparin immunoglobulin G (IgG)-enzyme immune assay (EIA) in patients with vaccine-induced immune thrombotic thrombocytopenia (VITT) with and without $100 \mathrm{IU} / \mathrm{mL}$ heparin. All VITT patients showed an enhanced binding which was significantly inhibited at high dose of heparin (100 IU/mL). (B) PF4-seroconversion after vaccination and severe SARS-CoV2 infection was followed up. IgG PF4/heparin antibody binding results in healthy volunteers before and after vaccination and COVID-19 patients in intensive care units showed four vaccinated volunteers displaying a positive EIA result after 7-14 days post-vaccination (red empty diamonds). OD: optical density.

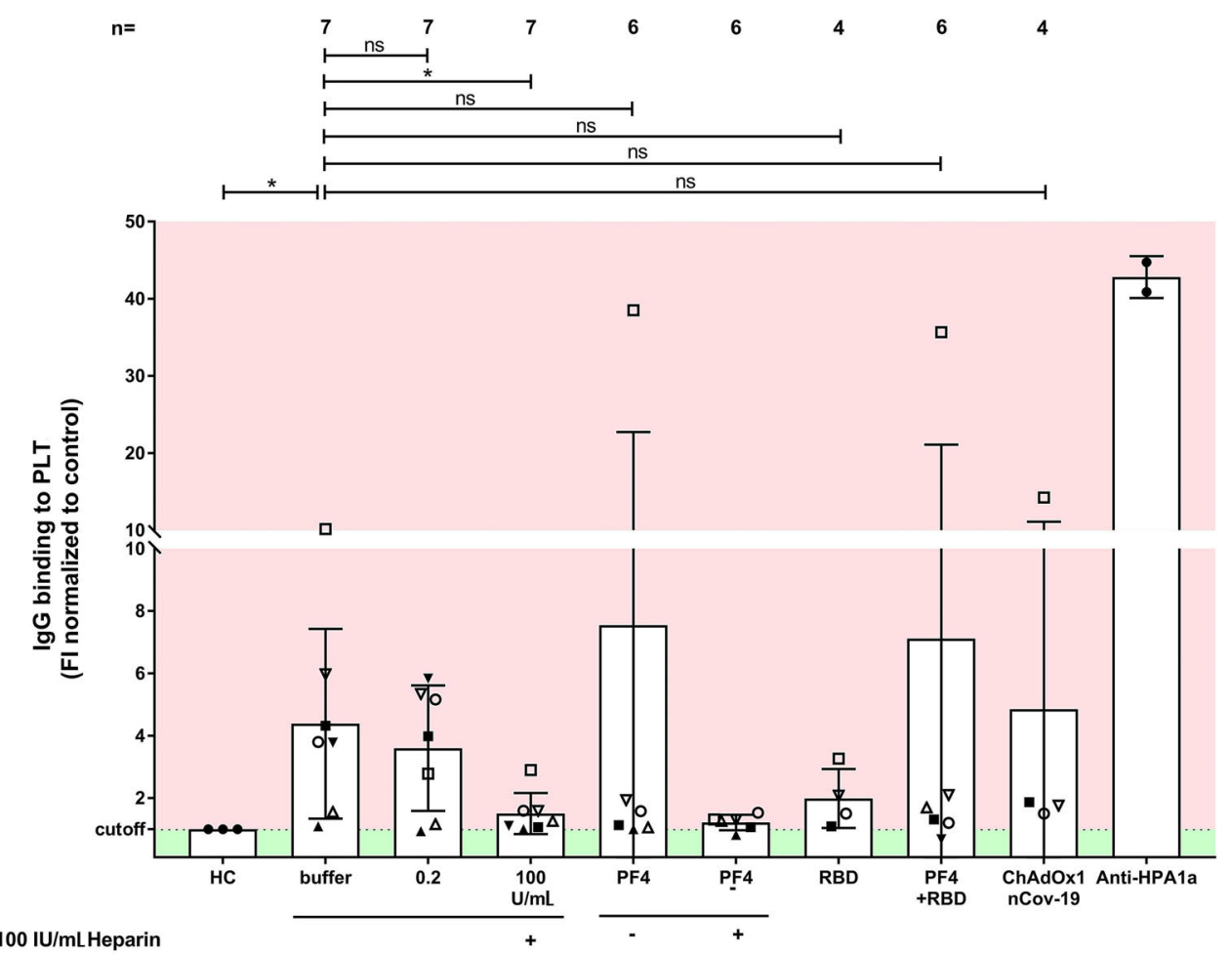

Figure 4. Immunoglobulin G binding to platelets by flow cytometry in sera of vaccine-induced immune thrombotic thrombocytopenia patients. Immunoglobulin $\mathrm{G}$ (IgG) binding to healthy washed platelets (PLT) after incubation with sera from vaccine-induced immune thrombotic thrombocytopenia (VITT) patients was measured (assessed by flow cytometry and expressed as fold increase (FI) normalized to controls). VITT patients showed significantly higher binding at the baseline in comparison to healthy controls, which was inhibited by high dose heparin. ns: not significant; $* P<0.05, \quad * * P<0.01$ $* * * P<0.001$ and $* * * * P<0.0001$. 
tively, $P<0.0001$, Figure $5 \mathrm{~A}$ ). On the other hand, sera from VITT patients showed slight but not significant binding to Spike-RBD (Online Supplementary Figure S2D). Most importantly, in the presence of PF4 the IgG binding was reduced when the concentration of RBD is increased above 6.5 $\mu \mathrm{g} / \mathrm{mL}$ (Figure 5A). However, sera from VITT patients did not show significant binding to S2 protein with and without PF4 (Figure 5B; Online Supplementary Figure S2E).

\section{Platelet activation in the heparin-induced platelet aggregation assay assay}

In order to investigate the ability of patients' sera to activate platelets, the HIPA assay was used with several modifications. Sera were incubated with washed platelets in the presence of i) buffer, ii) $0.2 \mathrm{IU} / \mathrm{mL}$ LMWH, iii) $100 \mathrm{IU} / \mathrm{mL}$ UFH, iv) an Fcy receptor IIa (Fc $\gamma$ RIIA)-blocking monoclonal antibody (mAb IV.3), v) $30 \mathrm{mg} / \mathrm{mL}$ IVIG, vi) $25 \mu \mathrm{g} / \mathrm{mL}$ PF4, vii) $50 \mu \mathrm{g} / \mathrm{mL}$ Spike$\mathrm{RBD}$, viii) PF4/Spike-RBD complexes, ix) PF4+RBD or $\mathrm{x}$ ) $\mathrm{ChAdOx} 1 \mathrm{nCoV}-19$. Conditions with PF4 and RBD were also repeated in the presence of high concentration of heparin $(100 \mathrm{IU} / \mathrm{mL}$ unfractionated heparin [UFH]). We observed platelet activation in the presence of buffer in eight of eight VITT patients (median time to platelet aggregation 5 minutes [min], no range 5-10 $\mathrm{min}$ [ $\mathrm{min}^{*}$, Figure $6 \mathrm{~A}$ ), but not in sera from vaccinated individuals
A

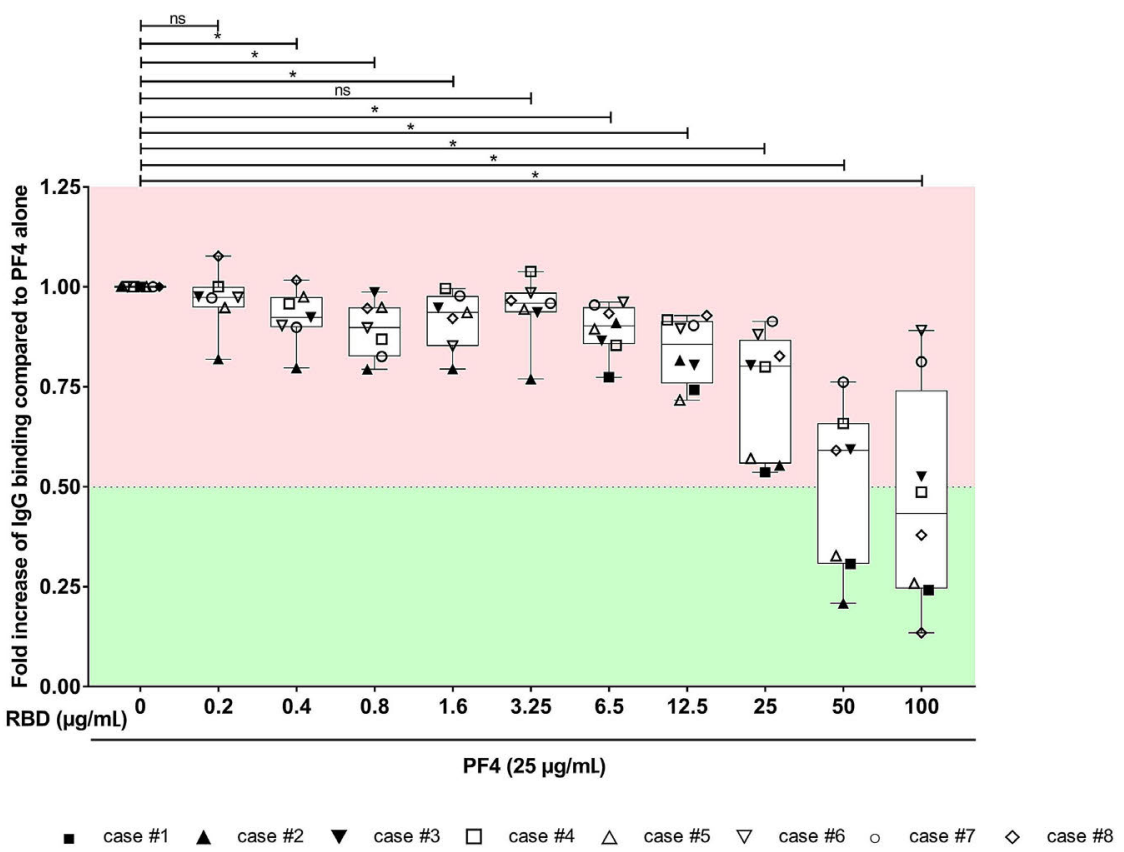

Figure 5. Immunoglobulin $\mathrm{G}$ binding to SARS-CoV-2 Spike-RBD and S2 in the presence and absence of PF4. (A) Immunoglobulin $\mathrm{G}$ (IgG) binding to SARSCoV-2 Spike-RBD was assessed by IgGenzyme immune assay (EIA) and expressed as fold increase to PF4 alone. (B) IgG binding to SARS-CoV-2 S2/PF4 complexes was assessed by EIA and expressed as fold increase to PF4 alone. ns: not significant; $* P<0.05, * * P<0.01$, $* * * P<0.001$ and $* * * * P<0.0001$.

B

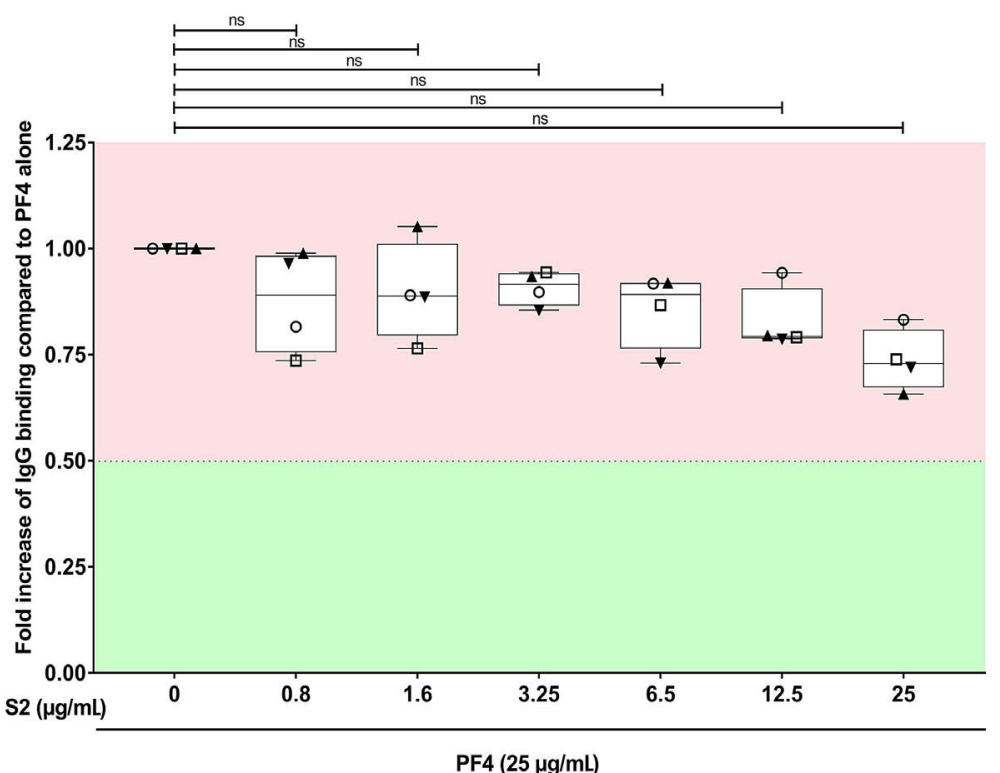


with anti-PF4 antibodies, who did not develop any clinical sign of thromboembolic complications, without side effects (Online Supplementary Figure S3A). Moreover, only one of the sera from patients with severe COVID-19 who were tested positive in PF4/heparin EIA showed platelet activation in the HIPA assay. (Online
Supplementary Figure S3B). Interestingly, the reaction was weaker in the presence of low molecular weight heparin (median time to aggregate: $5 \mathrm{~min}, 5-10 \mathrm{~min}$ [range] vs. 30 min, 5->45min [range], Figure $6 \mathrm{~A})$. All reactions were inhibited by a high dose of heparin $(P=0.008$, Figure $6 \mathrm{~A})$. In presence of PF4, sera from VITT patients showed

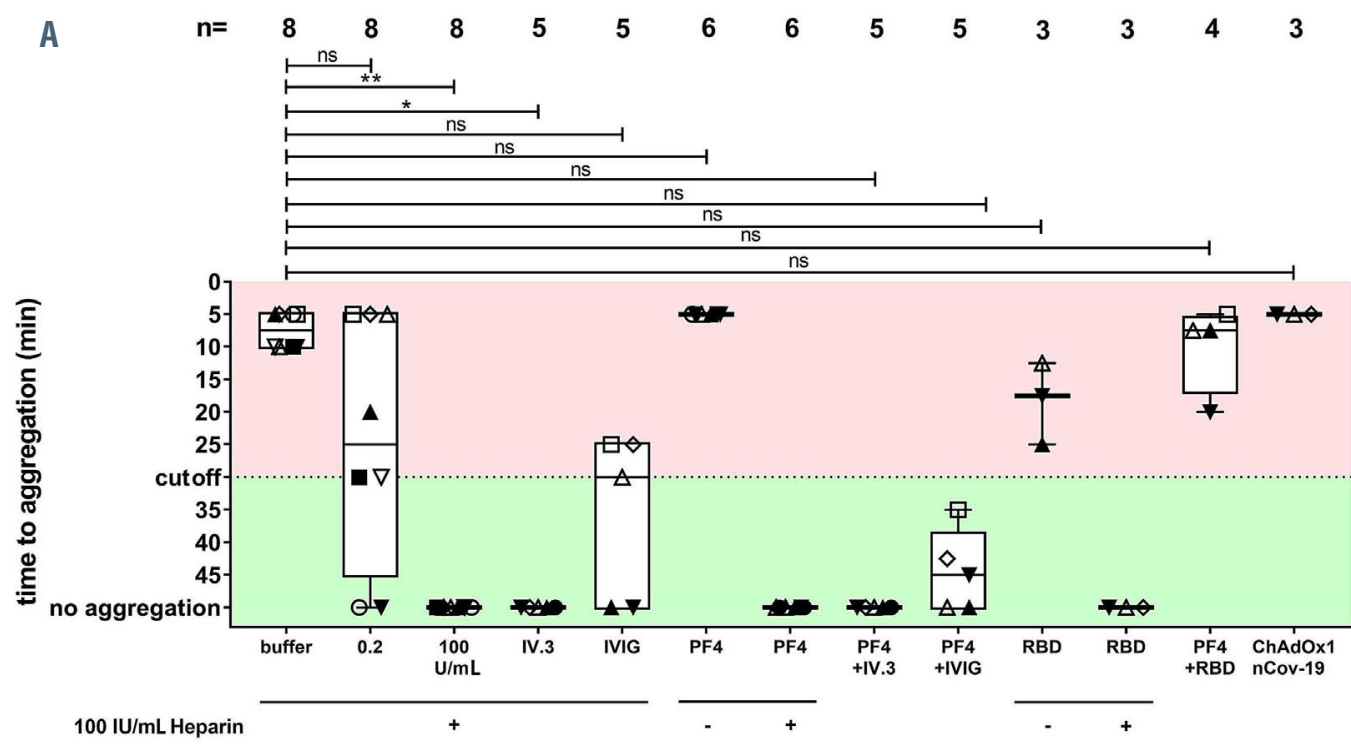

- case \#1 $\Delta \quad$ case \#2 $\boldsymbol{\nabla}$ case \#3 $\square$ case \#4 $\Delta$ case \#5 $\nabla$ case \#6 $\quad \circ$ case \#7 $\diamond \quad$ case \#8

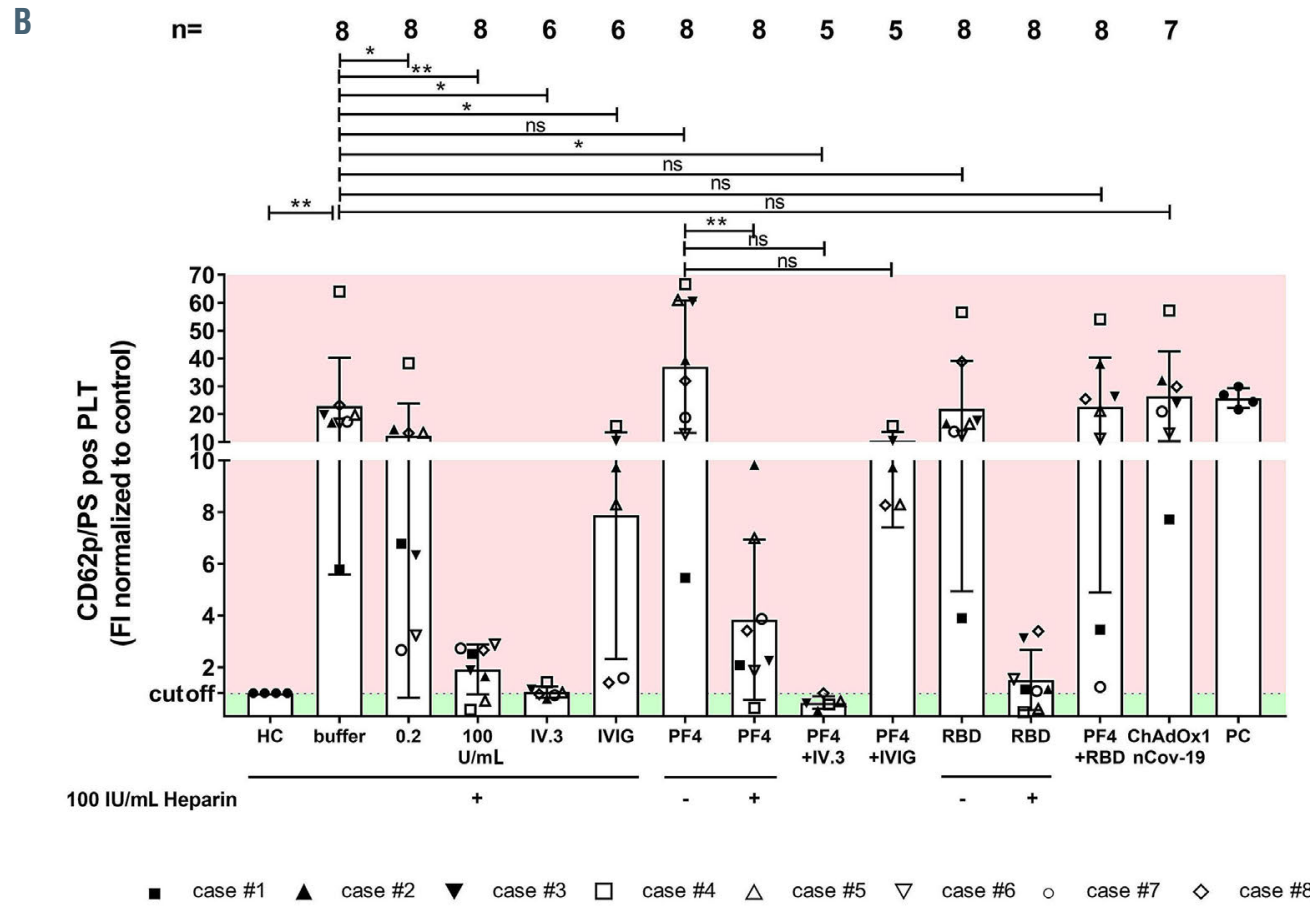

Figure 6. Antibody-mediated platelet activation and generation of procoagulant platelets. Results of the platelet activation assay (HIPA) with modifications in the vaccine-induced immune thrombotic thrombocytopenia (VITT) patients. Each dot represents the median of four different donors. (A) All VITT patients presented platelet (PLT) activation with buffer alone, which was significantly increased by PF4 but inhibited with high dose of heparin. Procoagulant platelets (CD62P/phosphatidylserine [PS] positive) in different settings were analyzed via Annexin V-FITC and CD62p-APC antibody staining. (B) Where indicated, platelets were treated with PF4, $0.2 \mathrm{U} / \mathrm{mL}$ and $100 \mathrm{IU} / \mathrm{mL}$ heparin, RBD and ChAdOx1 nCoV-19A. Data are presented as mean \pm standard deviation of the measured fold increase compared to control. ns: not significant; $* P<0.05, * * P<0.01, * * * P<0.001$ and $* * * * P<0.0001$. The number of sera tested is reported in each graph. Dotted lines represent the cutoffs determined testing sera from healthy donors. Fl: fold increase. 
strong platelet activation (median time to platelet aggregation $5 \mathrm{~min}, 5-5 \mathrm{~min}$ [range], Figure 6A). Most importantly, platelet activation was completely inhibited by the mAb IV.3 that blocks the FcyRIIa and by high doses of $\operatorname{IgG}(>45 \mathrm{~min}$, no aggregation, Figure 6A). No significant change was found when PF4/RBD complexes were added. Antibody-mediated platelet activation was inhibited when low molecular weight heparin (LMWH) was added at low concentrations by testing three sera. When sera from VITT patients were diluted, specific binding was observed to PF4 while no reaction in the buffer was found (Figure 7A).

\section{Sera of vaccine-induced immune thrombotic thrombo- cytopenia patients induce PF4-dependent procoagu- lant phenotype}

In order to explore the mechanism of coagulation dysregulation in VITT, sera were incubated with washed platelets from healthy donors in the presence of buffer, heparin, mAb IV.3, IVIG, PF4, PF4+IVIG, PF4+RBD, the Spike-RBD protein or the vaccine ChAdOx1 nCoV-19. FC analyses revealed that sera from VITT patients induce remarkable changes in the distribution of CD62p/PS positivity (FI CD62p/PS positive platelets [PLT]: 22.94 \pm 6.14 vs. $0.90 \pm 0.63$, respectively, $P=0.009$, Figure $6 \mathrm{~B}$, Online

A $n=$ 4 4 4 4 4

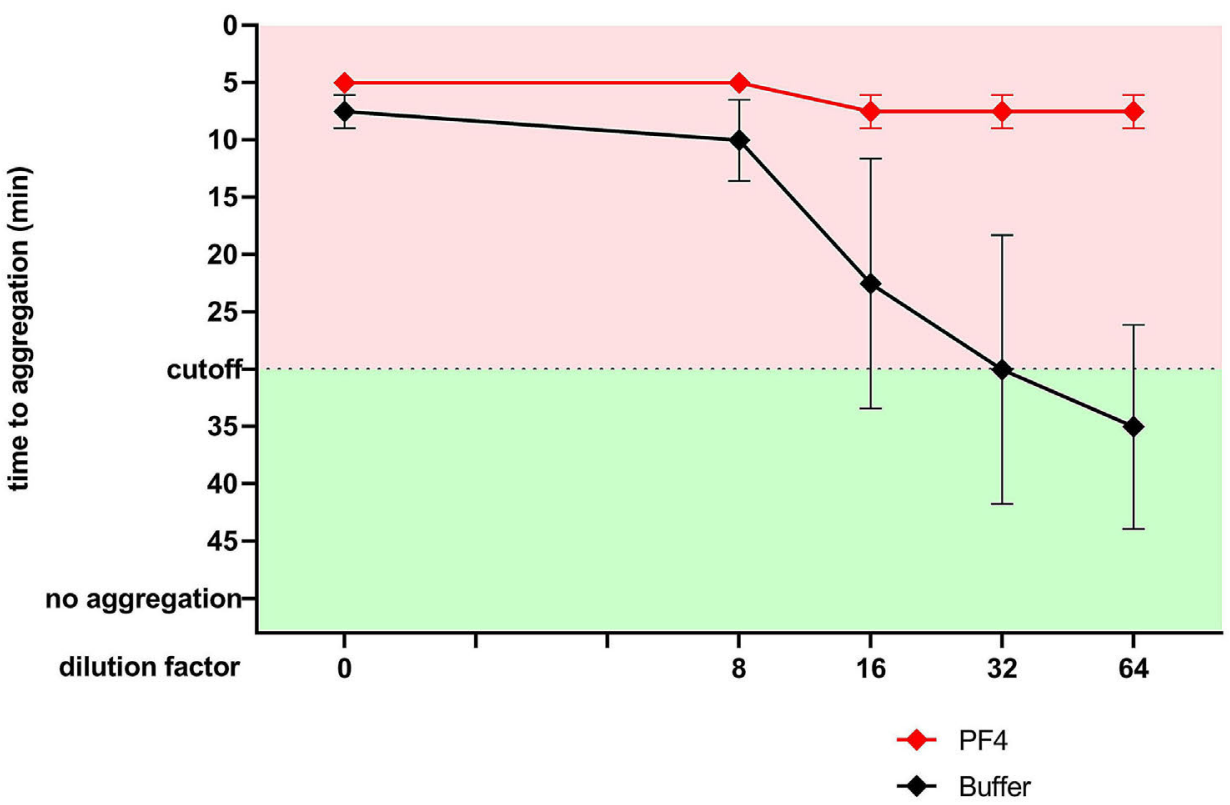

Figure 7. Antibody-mediated platelet activation and generation of procoagulant platelets with diluted sera. (A) Results of heparininduced platelet aggregation assay (HIPA) at different titrations of sera from vaccine-induced immune thrombotic thrombocytopenia (VITT) patients. Note that diluted sera (from 1:64) activated platelets only in the presence e of PF4. (B) Effect of sera from VITT patients at different titrations on the development of procoagulant platelets. Note that diluted sera (from 1:8) activated platelets only in the presence e of PF4. Data are presented as mean \pm standard deviation of the measured fold increase (FI) compared to control. ns: not significant; $\star P<0.05$; $* * P<0.01, \quad * * * P<0.001$ and $* * * * P<0.0001$. The number of sera tested is reported in each graph. Dotted lines represent the cutoffs determined testing sera from healthy donors.

B $\mathrm{n}=$ 4 4 4 4 4

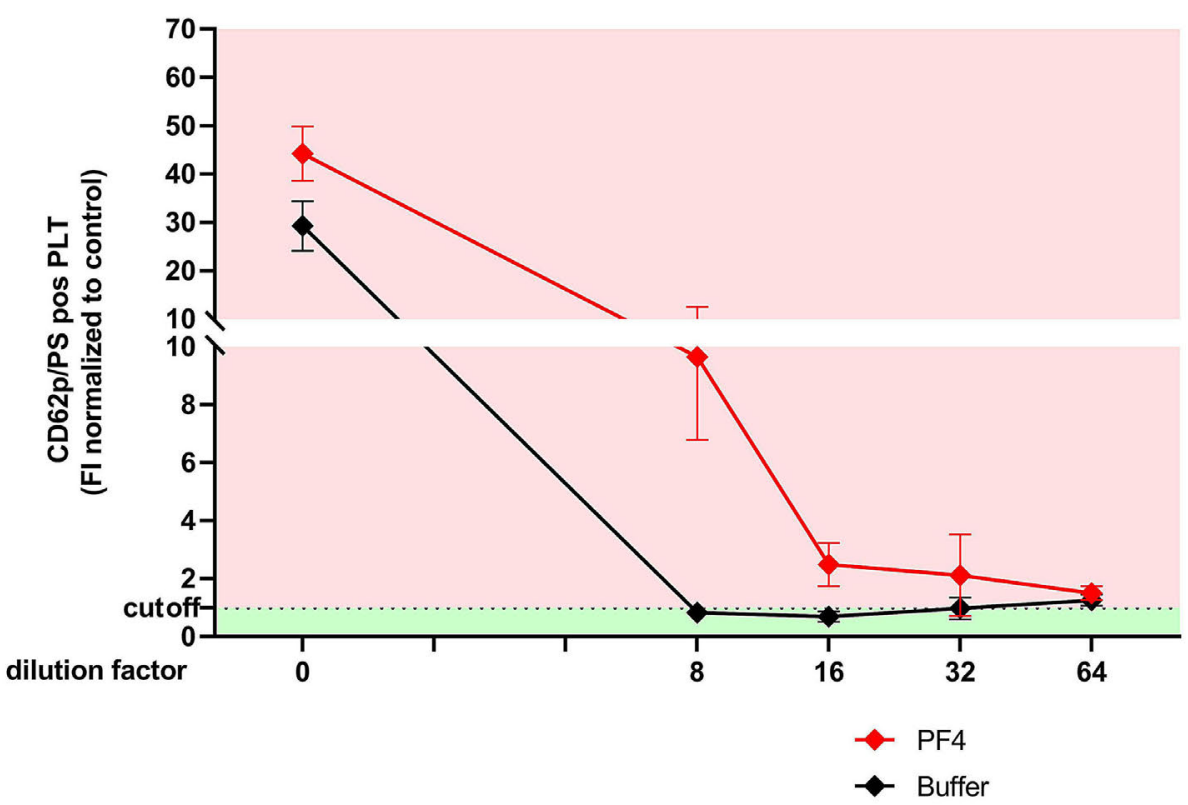


Supplementary Figure S4). In contrast, the platelet population was almost non-affected after incubation with sera from vaccinated controls (Online Supplementary Figure $S 5 A$ ). Interestingly, the generation of procoagulant platelets was reduced by $0.2 \mathrm{IU} / \mathrm{mL} \mathrm{LMWH}$ (FI CD62p/PS positive PLT: $13.32 \pm 11.50, \mathrm{P}=0.016$, Figure $6 \mathrm{~B}$ ) and completely inhibited by high concentration of UFH in VITT patients (FI CD62p/PS positive PLT: $1.92 \pm 0.96, \mathrm{P}=0.008$, Figure 6B, Online Supplementary Figure S4). These reactions were also inhibited by the FcrRIIA blocking with $\mathrm{mAb}$ IV.3 as well as by high concentrations of IgG (FI CD62p/PS positive PLT: $1.04 \pm 0.22, P=0.031$ and FI CD62p/PS positive PLT: $7.88 \pm 5.56, P=0.031$, respectively, Figure $6 \mathrm{~B}$ ). No significant increase of procoagulant platelets was also observed in presence of PF4 (FI CD62p/PS positive PLT: $37.07 \pm 23.73, P=0.078)$ and in the presence of Spike-RBD alone (FI CD62p/PS positive PLT: $22.02 \pm 17.09, P=0.195)$. While an increased generation of procoagulant platelets was observed after the incubation of sera from severe COVID-19 patients, no significant change was observed when sera from vaccinated individuals with anti-PF4 antibodies were tested (Online Supplementary Figure $S 5 A$ and $B$ ).

In order to identify the target antigen of the platelet activating antibodies, the HIPA and FC analyses were repeated at different titrations of sera from VITT patients. Interestingly, diluted sera (from 1:64) were able to activate platelets and induce a procoagulant phenotype only in the presence of PF4 (Figure 7A and B, respectively).

\section{Discussion}

The increasing number of reports on rare thrombotic events after SARS-CoV-2 vaccination draw public attention and led to concerns regarding the safety of this vaccine due to the uncertainty of the origin of these undesired reactions..$^{7.9}$ In order to understand the pathophysiology of this phenomenon, the so-called VITT, we analyzed sera from eight patients. Our mostly young, generally fit cohort of patients, presented acutely with atypical thrombosis, primarily, but not exclusively involving the cerebral venous sinuses, an extremely rare manifestation of thrombosis in the general population. All cases developed symptoms within 6-20 days after the ChAdOx1 nCoV-19 vaccination showing a temporal relationship between vaccination and symptoms. The main findings in these cases were thrombocytopenia, high D-dimer, low fibrinogen, and high-titer IgG antibodies against PF4 that can induce procoagulant platelet phenotype.

After intensive laboratory investigations of the VITT cases, we were able to identify the serological profile of the pathological antibodies. In a small cohort of vaccinated volunteers, approximately $10 \%$ of the individuals developed IgG antibodies against PF4/polyanion complexes within 14 days after the first vaccination; none of them had been exposed to heparin in the past 100 days. We observed that IgG binding to PF4 in these sera as well as in VITT sera can be inhibited by heparin but also by increasing the concentration of Spike-RBD. These data may suggest that these antibodies are specific for conformational changes in PF4 that might be induced by negatively charged structures. Of note, no significant IgG binding to platelets was observed in the presence of the vaccine ChAdOx1 nCoV-19. Accordingly, it is very unlikely that the vector (pCDNA4) may be responsible for the high PF4-seroconversion rate in vaccinated individuals. Comparable data were reported by Greinacher et al. ${ }^{7}$ and Schultz et al. ${ }^{9}$ in two very recent reports that appeared while our manuscript was in preparation. In addition to their observations, we were also able to demonstrate that sera from VITT patients directly induce procoagulant platelets, suggesting a possible mechanism for thrombotic events seen in patients with VITT. This is further corroborated by the pathological studies in two of our patients. Despite the distinct immediate causes of death in these two fatal cases, namely fatal cerebral sinus thrombosis and intracerebral hemorrhage, the two autopsy reports showed striking similarities. In addition to arterial, arteriolar and venous thrombosis in various organs and pulmonary thromboembolism, both cases showed a striking occlusion of multiple glomeruli and afferent arterioles by hyaline thrombi composed of fibrin and platelets, but lacking erythrocytes. The kidney morphology bears resemblance to thrombotic microangiopathy, but we failed to identify erythrocyte fragmentation, a key feature of thrombotic microangiopathy. ${ }^{10}$ Both patients, however, had normal kidney function (highest creatinine level 0.5 $\mathrm{mg} / \mathrm{dL}$ in case \#2 and $0.8 \mathrm{mg} / \mathrm{dL}$ in case\#3) until briefly before death, indicating rapid pre-terminal development of glomerular microthrombosis. White thrombi have been associated with antibody-mediated platelet activation. ${ }^{10,11}$

Our data indicate that IgG antibodies against PF4 increase the generation of procoagulant platelets in VITT. However, we cannot exclude other co-factor(s) that could also induce thromboembolic complications in vivo. We report on VITT after $\mathrm{ChAdOx} 1 \mathrm{nCoV}-19$, which is the only SARS-CoV-2 vaccine that includes a simian adenovirus. Disturbances of platelets have been described in association with the intravenous administration of adenovirus gene therapy vectors although it is unclear how that might relate to isolated thrombocytopenia as an adverse event of the vaccine.

Finally, the observed clinical and laboratory features of the VITT are exceptional and rare. Therefore, the value of the COVID-19 vaccination to provide critical protection should be considered higher compared to the significant health risk of COVID-19. With the better recognition of this rare complication and the availability of efficient therapies, the risk-benefit ratio of $\mathrm{ChAdOx} 1 \mathrm{nCoV}-19$ might be further reconsidered.

\section{Conclusion}

Although the incidence of VITT after ChAdOx1 nCoV19 vaccination is very low, the mortality rate is high (37.5\% in our case series). Since a global vaccination campaign is underway and large numbers of people will be vaccinated, an increase in the number of people with this side effect is to be expected, highlighting the importance of a better understanding of the pathophysiology of VITT. In this study, we present immunological and pathological findings in patients with VITT. Furthermore, we show the contribution of antibody-mediated platelet activation in the pathogenesis of VITT.

\section{Disclosures}

No conflicts of interest to disclose.

\section{Contributions}

$K A, P M, U J S, F F$, and TB designed the study; JS, MG, MP, 
$M B, A B, H B, F D, A V, H H, N H, K K, C L, B L, J M, M M, S N, C S$, $B P, U J S, M W$ and GCP were responsible for the treatment of patients and collected and analyzed the clinical data; TB, GU, KA and FF reviewed medical reports; KA, GU,JAM, AS and LP performed the experiments; WAW and DV analyzed the radiological images; $H B, V W, F F$, and $P M$ analyzed the autopsy findings and performed tissue studies; $H J$ produced Spike protein; KA, LP, AS, $G U, N S, F F$ and TB analyzed the data, interpreted the results and wrote the manuscript. All authors read and approved the manuscript.

\section{Acknowledgments}

We thank our technicians Karoline Weich, Simone Weit,
Franziska Lyshy, Marco Mikus and Flavianna Rigoni for their excellent technical support. We also thank Harald Klüter and Martin Holderried for vaccines.

\section{Funding}

This work was supported by grants from the German Research Foundation and from the Herzstiftung to TB (BA5158/4 and TSG-Study), by special funds from the state of Baden-Württemberg for autopsy-based COVID-19 research and the DEFEAT PANDEMIcs network funded by the BMBF to $P M$ and FF. JAM is supported by a grant from the German Research Foundation.

\section{References}

1. Grasselli G, Greco M, Zanella A, et al. Risk factors associated with mortality among patients with COVID-19 in intensive care units in Lombardy, Italy. JAMA Intern Med. 2020;180(10):1345-1355.

2. Castells MC, Phillips EJ. Maintaining safety with SARS-CoV-2 vaccines. N Engl J Med. 2021;384(7):643-649.

3. Althaus K, Marini I, Zlamal J, et al. Antibody-induced procoagulant platelets in severe COVID-19 infection. Blood. 2021; 137(8):1061-1071.

4. Wolf ME, Luz B, Niehaus L, Bhogal P, Bazner $\mathrm{H}$, Henkes H. Thrombocytopenia and intracranial venous sinus thrombosis after "COVID-19 vaccine AstraZeneca"
Exposure. J Clin Med. 2021;10(8):1599.

5. Becker M, Strengert M, Junker D, et al. Exploring beyond clinical routine SARS CoV-2 serology using MultiCoV-Ab to evaluate endemic coronavirus cross-reactivity. Nat Commun. 2021;12(1):1152.

6. Greinacher A, Michels I, Kiefel V, MuellerEckhardt C. A rapid and sensitive test for diagnosing heparin-associated thrombocytopenia. Thromb Haemost. 1991;66(6):734 736.

7. Greinacher A, Thiele T, Warkentin TE, Weisser K, Kyrle PA, Eichinger S Thrombotic thrombocytopenia after ChAdOx1 nCov-19 vaccination. N Engl J Med. 2021;384(22):2092-2101.

8. Othman M, Labelle A, Mazzetti I, Elbatarny HS, Lillicrap D. Adenovirus-induced throm- bocytopenia: the role of von Willebrand factor and P-selectin in mediating accelerated platelet clearance. Blood. 2007;109(7):28322839

9. Schultz NH, Sorvoll IH, Michelsen AE, et al. Thrombosis and Thrombocytopenia after ChAdOx1 nCoV-19 Vaccination. N Engl J Med. 2021;384(22):2124-2130.

10. Brocklebank V, Wood KM, Kavanagh D. Thrombotic Microangiopathy and the Kidney. Clin J Am Soc Nephrol. 2018;13(2): 300-317.

11. Pierangeli SS, Espinola RG, Liu X, Harris EN Thrombogenic effects of antiphospholipid antibodies are mediated by intercellular cell adhesion molecule-1, vascular cell adhesion molecule-1, and P-selectin. Circ Res. 2001;88 (2):245-250. 